\title{
Persistent Infectious Diarrhea as a Risk Factor for Deterioration of Renal Function in Kidney Transplant Recipients
}

Authors:

Hue Yu Wang ${ }^{1,2}$

Yu-Tung Feng ${ }^{3}$

Ya-Ting Kung ${ }^{3}$

Ming-Kung Yeh ${ }^{4,5}$

Yaw-Bin Huang ${ }^{2}$

1 Department of Pharmacy, ChiMei Medical Center, Tainan, Taiwan.

2 College of Pharmacy, Kaohsiung Medical University, Kaohsiung, Taiwan.

3 Graduate Institute of Healthcare Administration and Medical Informatics, Kaohsiung Medical University, Kaohsiung, Taiwan.

4 Administration and Quality Assurance Center, Kaohsiung Medical University Hospital, Kaohsiung, Taiwan

5 School of Pharmacy, Graduate Institute of Medical Science, National Defense Medical Center, Taipei, Taiwan

6 Taipei Hospital, Ministry of Health and Welfare, Taipei, Taiwan

\section{Corresponding author:}

Hue Yu Wang.

Department of Pharmacy, Chi-Mei Medical Center, Tainan, Taiwan.

No.901, Zhonghua Rd., Yongkang Dist., Tainan City 710, Taiwan

E-mail: cmh5500wang@gmail.com

Keywords: Diarrhea, kidney transplantation, immunosuppressive, infection, mycophenolate

\begin{abstract}
Background: Gastrointestinal complications are common in kidney transplant recipients. Severe diarrhea might result in graft dysfunction or rejection if being ignored. The study aim was to describe the relationship between kidney allograft function and diarrhea, and to determine how diarrhea is influenced by various immunosuppressive regimens at a single center in Taiwan.
\end{abstract}

Methods: This was a retrospective and comparative study. We included kidney transplant recipients who suffered from persistent diarrhea between January 2009 and August 2015. Survival and regression analyses and sub-analyses of creatinine levels over different diarrhea periods were performed.

Results: We eventually collected 62 patients for analyses, including 16 with infectious diarrhea and 46 with noninfectious diarrhea. At $3^{\text {rd }}$ year post transplantation, patients with infectious diarrhea demonstrated a significantly lower cumulative renal function rate $(56.3 \%$ vs. $84.7 \%$, p < 0.05$)$. No significant differences were found between recipients receiving different immunosuppressive regimens in terms of renal function. However, recipients receiving mammalian target of rapamycin (mTOR) inhibitor and mycophenolate mofetil (MMF) tended to show a slower rate for deterioration of renal function.

Conclusion: In this study, infectious diarrhea, especially when persisting for more than 10.5 days, does significant damages to renal function and predicts for deterioration of renal function. Besides, regimen of mTOR inhibitor and MMF showed a trend toward reducing incidence of deterioration of renal function. Better understanding of the diarrhea etiologies to provide timely treatment for this illness and appropriate choice of immunosuppressive regimen might help maintain renal function for kidney transplant recipients. 


\section{Introduction}

New immunosuppressive agents have been introduced in clinical practice to prevent acute rejection episodes in kidney transplant recipients, however, drug-related adverse events such as infection, anemia, and diarrhea are still encountered frequently [1, 2]. Many studies have precipitated the use of immunosuppressive agents a strong risk factor for medication infections which lead to diarrhea [3-7]. Severe diarrhea might lead to weight loss, dehydration, increase of serum creatinine, and the fluctuating of immunosuppressive agent levels [8-10]. Moreover, it might result in graft dysfunction or rejection if being ignored $[6,10]$.

Mycophenolate mofetil (MMF) is one of the key components of the current immunosuppression regimen for patients who receive kidney transplantation surgery to prevent organ rejection [11], and its combinations with cyclosporine (CsA), tacrolimus (TAC), or mammalian target of rapamycin (mTOR) inhibitor are frequently prescribed. However, MMF is reported to be associated with an increased risk of both infectious and noninfectious diarrhea, which may link to poor renal allograft survival $[7,12,13]$.

Although severe diarrhea is thought to threaten the graft function and even survival, the studies for their correlation seem inconsiderable. The aim of this study was to describe the relationship between kidney allograft function and diarrhea, and to determine how diarrhea is influenced by various immunosuppressive regimens at a single center in Taiwan.

\section{Materials and Methods}

This was a retrospective, comparative study, conducted among kidney transplant recipients, who were defined as adult males or non-pregnant females undergoing primary deceased donor, living-unrelated or human leukocyte antigen-mismatched livingrelated donor kidney transplantations. A hospital multidimensional database management system that provides a 
platform upon which clinical professionals can build storage and analytic applications of all patients' data was used. Patient data included personal demographic information, visit of outpatient clinics, results of chemical/physical examinations, diagnoses, medication, hospitalization, and various sorts of medical expenses. After approved by the hospital ethics committee, we accessed to obtain data with adequate anonymity for further analyses.

Subjects were collected by the search of ICD-9 CM (the diagnosis International Classification of Diseases, Ninth Revision, Clinical Modification) codes such as 42.0 for "renal transplantation; kidney replaced by transplant" $\geq 1$ year. Medical records of these kidney transplant recipients who came for diarrhea at our transplant center between January 2009 and August 2015 were further analyzed. Diarrhea in the kidney transplant recipients was defined in the study as: (1) occurring 1 year after transplantation; (2) $\geq 3$ times/day and persisting for over 3 days; (3) diagnosed as "specified infectious cause: 001.x, 004.1, and 008.xx", "other and unspecified infectious diarrhea: 009.x", “other noninfectious causes: 558.2 and 558.3" and "other and unspecified diarrhea: 558.9, 787.91, and 564.5". The patients who were diagnosed with ICD-9 CM codes of 001.x, 004.1, 008.xx, and 009.x or those who received antimicrobial agents for diarrhea treatment were grouped as "Infectious diarrhea" (ID group), and those who were diagnosed with ICD-9 CM codes of 558.2, 558.3, 558.9, 787.91, and 564.5 and those who didn't receive anti-microbial agents were grouped as "Non-infectious diarrhea" (NID group). No fever, change of white blood counts, or use of antibiotic agents were confirmed with NID patients.

Clinical data included patient information of age, gender, diarrhea period, time from transplantation to onset of diarrhea, serum creatinine (Scr) of 1 month before diarrhea, Scr during diarrhea, change of Scr between 1-month 
before and during diarrhea (Scr, change), and the use of immunosuppressive agents, e.g. mM group (mTOR inhibitor-MMF), TM group (TAC-MMF), and CM group (CsA-MMF). The clinical features of patients receiving different immunosuppressive regimens were compared. The clinical features of patients with ID were also compared with those of the recipients with NID. The causes and the risk factors for deterioration of renal function after transplantation were also evaluated.

Statistical analyses were performed with the SPSS 10.0 statistical package. Descriptive statistics were calculated as mean \pm standard deviation for continuous variables, number and percentage for categorized variables. For comparison of continuous variables between groups, student's t-test was used. For comparison between groups of dichotomous variables, Chi-square test was used. Receiver operating characteristic curve was used to decide cutoff for diarrhea period. Survival analysis was done with the Kaplan-Meir method and the log-rank test. Cox regression was used to predict for renal dysfunction. A $p$ value of less than 0.05 was regarded as significant.

\section{Results}

Study flowchart was shown in Figure 1. Between January $1^{\text {st }} 2009$ and August $31^{\text {th }} 2015$, a total of 189 kidney transplant recipients who met the inclusion criteria were screened during the study period, including 115 patients with confirmed diagnosis of diarrhea (including infectious causes, non-infectious causes, and other unspecified diarrhea) and 74 patients with prescription of anti-microbial agents $\geq 3$ days. Excluding those with duplicated entry, transplantation $\leq 1$ year, diarrhea after graft failure and missing data, 62 patients were then collected for further analyses. Among them, 16 were classified as ID and 46 were NID.

The clinical characteristics of these patients are shown in Table 1. Among the 62 patients collected for the study, 38 $(61.3 \%)$ were female and the mean age at 
the time of persistent diarrhea 46.4 years. The average time from transplantation to a persistent diarrhea was $6.4 \pm 4.5$ years. There were $16 \quad(25.81 \%)$ patients presenting with ID, including $3(18.75 \%)$ for viral infections (cytomegalovirus (CMV) for 2 and rotavirus for 1) and 13 for bacterial infections (Clostridium difficile for 8, Salmonella spp. for 3, and pathogens unidentified for 2). In addition, 46 patients $(74.19 \%)$ were presented with NID, containing noninfectious colitis for 14 , watery diarrhea (> 3 time/day for $3 \sim 5$ days) for 9, drug-related colitis for 3, functional diarrhea for 1 , and other and unspecified diarrhea for 19. Three main combinations of immunosuppressive treatments were used in the study: 1) mammalian target of rapamycin inhibitor (m-TORI) and MMF; 2) TAC and MMF; and 3) CsA and MMF.

A cutoff value of 10.5 days for diarrhea period was obtained according to Youden's index, offering definition for a "persistent diarrhea" in our study. Among the study population, $18(29.0 \%)$ patients had persistent diarrhea, and they had significantly prolonged elevation of serum creatinine $(0.52 \pm 0.52$ vs. $0.06 \pm 0.22$ $\mathrm{mg} / \mathrm{dL} ; \mathrm{p}<0.001)$.

When types of diarrhea were concerned, both ID and NID groups experienced renal dysfunction in a comparable way. However at $3^{\text {rd }}$ year post transplantation, the ID group demonstrated a significantly lower cumulative renal function rate $(56.3 \%$ vs. $84.7 \%, p<0.05$; Table 2). Nevertheless, we found no significant evidence of a consistent negative slope for the deterioration of renal function (Figure 2).

No matter which immunosuppressive combination the patients received in the study, they more or less suffered from diarrhea and their renal function deteriorated in a comparable way. No significant differences were found as for cumulative renal function rate and mean or median renal function year (Table 3). However, the $\mathrm{mM}$ group tended to show a slower deterioration rate for renal function (Figure 3). 
When multivariate analyses were performed to find out risk factors for the deterioration of renal function for study population, ID patients possessed a 3.24 times $(p=0.041)$ of risk for deterioration of renal function when compared with NID patients in the study (Table 4).

\section{Discussion}

With reported incidences as high as $64 \%$ in large clinical trials, diarrhea occurs commonly following kidney transplantation and is frequently caused by infections and immunosuppressive medications $[6,14]$. Leading to water and electrolyte disturbances, interfering with the absorption of nutrients and drugs, and thus negatively affecting the quality of life of the patients, post-transplantation diarrhea might sometimes persist for a long period of time, compound the health status of the patients, and even decrease the graft survival $[15,16]$. In our study, infectious etiologies accounted for only $25.8 \%$ of the causes of diarrhea among kidney transplant recipients and the average time from transplantation to a persistent diarrhea was $6.4 \pm 4.5$ years. These findings were somewhat different from what Bamias et al have published, which indicates infection is usually the cause of diarrhea after 4-year posttransplantation [14]. However, our findings were similar with the results of several previously published studies which did not take into account the severity and duration of the diarrhea [ 9 , 16-18]. Patients' characteristics might be the main explanation for the similarity; patients of our study were all collected from out-patient and emergency department (diarrhea $<10.5$ days for $71.0 \%$ of the patients) and preliminary supportive treatments were readily offered. Their final diagnoses were not surely confirmed because fecal specimen collection and examination were not even done.

Renal dysfunction is common in liver transplant patients [19]; however, the published incidence of deterioration of renal function and its association with diarrhea in kidney transplant patients 
seem limited. In this study, serum creatinine change became significant when the diarrhea period persisted for more than 10.5 days $(0.52 \pm 0.52$ vs. 0.06 $\pm 0.22 \mathrm{mg} / \mathrm{dL} ; \mathrm{p}<0.001)$, indicating the impairment of renal function became greater and irreversible damage to graft might occur as well [10].

With limited number of ID cases in our study, bacteria were found the most common etiologies, and the most common cause is Clostridium difficile infection (8 out of 62 patients; $12.90 \%$ ). The findings were similar to previous published data for both causative pathogen and occurrence rate [12, 20], although we had a relatively small sample size for our study. CMV is a reported frequent cause of diarrhea in the organ recipients, which demonstrates significant deleterious effects on the clinical course of the graft [20]. Retrospective analyses for the 2 diarrhea cases due to CMV infection showed that their diarrhea period exceeded 20 days though valganciclovir was offered timely and their renal function had significantly deteriorated accordingly.

Although patients in both ID and NID groups demonstrated renal function impairment in a comparable way, the ID group showed a significantly lower cumulative renal function rate at $3^{\text {rd }}$ year post transplantation. Together with the results of multivariate analyses that ID patients had a 3.24 times $(p=0.041)$ of risk for deterioration of renal function when compared with NID patients, a negative long-term effect of infectious diarrhea to the renal function of kidney transplant recipients was found. The mechanism underlying infectious diarrhea impairs renal function must be elucidated; studies with larger scale are thus warranted.

The relatively high occurrence rate of $36.1 \%$ of non-infectious gastrointestinal adverse effects is a major concern when physicians prescribe MMF, although its adverse effect profile is comparatively mild [6, 21, 22]. Diarrhea caused by MMF is frequently dose-related, dosage reduction or the complete 
withdrawal of MMF reduces the occurrence of gastrointestinal adverse effects [22, 23]. In our study, MMF was offered with daily dose of 0.5 to $1.0 \mathrm{gm}$, which was far lower than the defined daily dose of $2.0 \mathrm{gm}$. The relatively low dose we used for MMF might be the explanation for the short period of diarrhea $(71.0 \%$ of the patients with diarrhea of $\leq 10.5$ days). However, NID accounted for $74.2 \%$ of the diarrhea cases, and we attributed this to patients' characteristics in the study.

In this study, renal function deteriorated in a comparable way as posttransplant diarrhea occurred no matter which immunosuppressive combination the patients had received. No significant differences of cumulative renal function rate and mean or median renal function year between different immunosuppressive combinations were found. However, patients in the $\mathrm{mM}$ group tended to show a slower deterioration rate for renal function, indicating less incident renal dysfunction was observed and a benefit was maintained throughout the study period. This might be attributed to the replacement of CNIs by mTOR inhibitor, which may prevent renal impairment in organ transplantation recipients [24].

Our study has several methodological aspects to be considered. First, study sample size may have been too small, particularly with the ID patient number and patients with persistent diarrhea defined in the study. However, after different statistic methods were used, significant deterioration of renal function was found in the group with diarrhea above 10.5 days. Large variance of change in serum creatinine was found in small size of study sample indicates correlation between diarrhea length and renal function. Small sample size of the study may reduce the generalization of study results; the study still gives credit to the relation between diarrhea type and/or length and renal function. Secondly, $\mathrm{m}$-TOR inhibitor is recently introduced to Taiwan, and again, the number of patients 
receiving combination of m-TOR inhibitor and MMF is limited when compared to those receiving combination of CNI and MMF. However, interestingly, the tendency of m-TOR inhibitor to protect renal function was shown in the study. Finally, the dosage of immunosuppressive agents used may differ from transplantation center to others. This might lead to different ID incidences in post renal transplantation population. It should be noted that ID represented only $25.8 \%$ of diarrhea type in our study. Therefore, our data may not be easily extrapolated.

\section{Conclusion}

Our study brings new data in the direction showing that infectious diarrhea does more damages to renal function than non-infectious ones in kidney transplant recipients, especially when the diarrhea persists for more than 10.5 days. Besides, the combination of MMF and mTOR inhibitor showed a trend toward reducing incidence of deterioration of renal function in kidney transplant recipients. Better understanding of the diarrhea etiologies to provide timely treatment for this illness and appropriate choice of immunosuppressive regimen might help maintain renal function for kidney transplant recipients.

\section{Acknowledgement}

The authors would like to express their gratitude to Dr. Richard H. Davis, Ph.D., English, as a second language teacher for his gratuitous assistance of English grammar examining and editing. The study was approved by the Institutional Review Board (ChiMei Medical Center No. 10002-010). 
Medical Research Archives, Vol. 4, Issue 7, November 2016

Persistent Diarrhea Impairs Renal Function

\section{Figures}

\begin{tabular}{|c|c|c|c|}
\hline \multicolumn{4}{|l|}{ Figure 1. Study flowchart } \\
\hline & $\begin{array}{r}\text { ICD-9 CD } \\
\text { Renal tr: } \\
\text { (Kidney repla } \\
\text { n }\end{array}$ & $\begin{array}{l}\text { ode: } 42.0 ; \\
\text { lantation } \\
\text { by transplant) } \\
9\end{array}$ & \\
\hline \multicolumn{2}{|c|}{$\begin{array}{l}\text { ICD-9 CM codes for diarrhea: } \\
\text { 1. } 001 . x, 004.1 \text {, and } 008 . x x \text { (specified infectious cause) } \\
\text { 2. } 009 . x \text { (other and unspecified infectious diarrhea) } \\
\text { 3. } 558.2,558.3 \text { (other noninfectious causes) } \\
\text { 4. } 558.9,787.91,564.5 \text { (other and unspecified diarrhea) } \\
n=115\end{array}$} & & $\begin{array}{l}\text { Anti-diarrhea agents }(\geq 3 \text { days }) \\
\text { 1. Kaolin suspension } \\
\text { 2. Loperamide } \\
\text { 3. Cholestyramine } \\
\text { 4. Lactoacillus bifidus } \\
\mathrm{n}=\mathbf{7 4}\end{array}$ \\
\hline \multicolumn{4}{|c|}{$\begin{array}{l}\text { Excluded (127): } \\
\text { Duplicate (54) } \\
\text { Post-transplant } \leq 1 \text { year (2) } \\
\text { Graft failure (1) } \\
\text { Incomplete data (70) } \\
\mathbf{N}=62\end{array}$} \\
\hline & $\begin{array}{l}\text { Infectious diarrhea } \\
\qquad \mathrm{n}=16\end{array}$ & \multicolumn{2}{|c|}{$\begin{array}{l}\text { Non-Infectious diarrhea } \\
\qquad n=46\end{array}$} \\
\hline $\begin{array}{c}\text { Bacteria } \\
n=13\end{array}$ & $\begin{array}{c}\text { Viruses } \\
\mathbf{n}=3\end{array}$ & & \\
\hline
\end{tabular}


Figure 2. Cumulative renal function rate for patients with different types of diarrhea at different diarrhea periods after kidney transplant

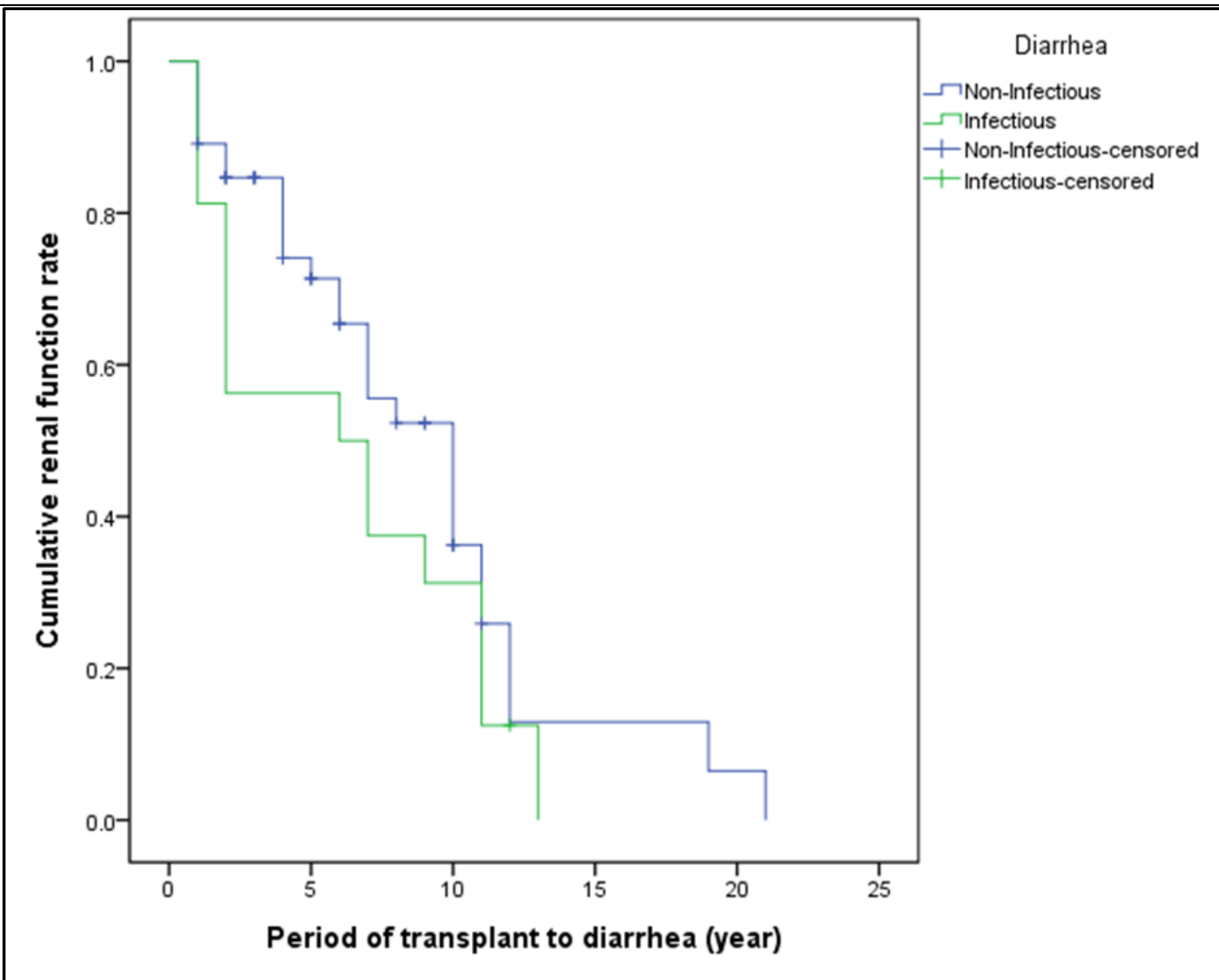


Figure 3. Cumulative renal function rate for patients with different immunosuppressive therapies at different periods after kidney transplant

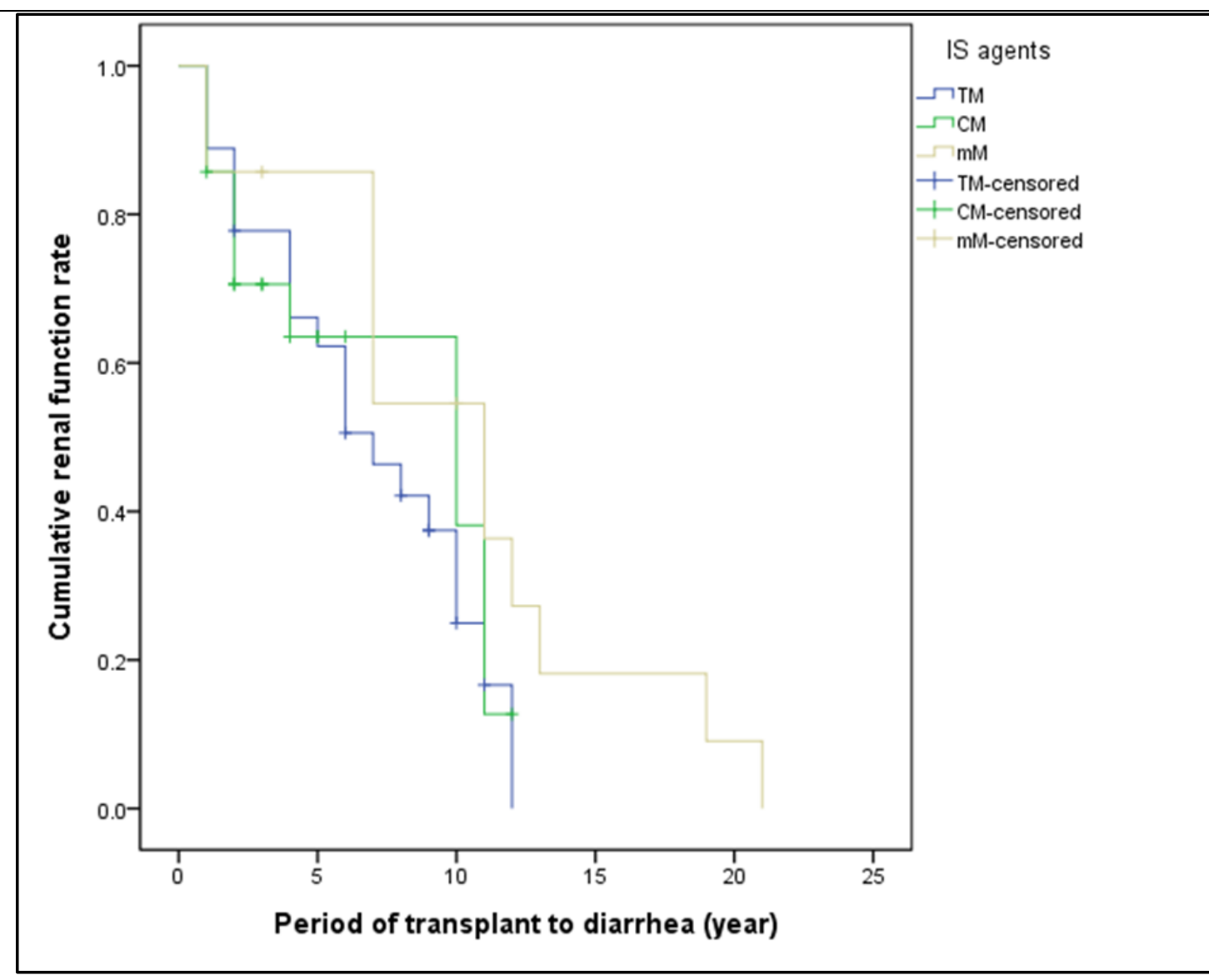

IS agents: Immunosuppressive agents. 


\section{Tables}

Table 1. Patient demographics and characteristics $(\mathrm{N}=62)$

\begin{tabular}{lcc}
\hline & Frequency & Percent \\
\hline Age (mean, SD) & 46.4 & 13.3 \\
Time: transplant to diarrhea (year) & 6.4 & 4.5 \\
Gender & & \\
$\quad$ Male & 24 & 38.7 \\
$\quad$ Female & 38 & 61.3 \\
Diarrhea & & \\
$\quad$ Non-Infectious & 46 & 74.2 \\
$\quad$ Infectious & 16 & 25.8 \\
Immunosuppressive agents & & \\
CM (CsA-MMF) & 21 & 33.9 \\
$\quad$ TM (TAC-MMF) & 27 & 43.5 \\
$\quad$ mM (mTOR inhibitor-MMF) & 14 & 22.6 \\
Scr, change (mg/dL; mean, SD) & & \\
$\quad$ Diarrhea $\leq 10.5$ days & $0.06^{*}$ & 0.22 \\
$\quad$ Diarrhea > 10.5 days & $0.52^{*}$ & 0.52 \\
Scr, status & & \\
$\quad$ Decreased or no change & 19 & 30.6 \\
$\quad$ Increased & 43 & 69.4 \\
Diarrhea day (mean, SD) & 11.92 & 14.18 \\
$\quad$ Median & 5 & \\
$\quad$ Min.-Max. & 2 & 68 \\
Diarrhea period & & \\
$\quad \leq 10.5$ days & 44 & 71.0 \\
$\quad>10.5$ days & 18 & 29.0 \\
I & &
\end{tabular}

Immunosuppressive agents: C: cyclosporine (CsA); m: mammalian target of rapamycin inhibitor (mTOR inhibitor); M: Mycophenolate mofitil (MMF); T: Tacrolimus (TAC). Scr: Serum creatinine; Scr, change: change of Scr between 1-month before and during diarrhea.

$* p<0.001$ 
Table 2. Comparison of diarrhea etiologies for cumulative renal function rate at different diarrhea periods after kidney transplant

\begin{tabular}{|c|c|c|c|c|c|c|c|c|c|c|c|}
\hline \multirow[t]{2}{*}{ Diarrhea } & \multicolumn{6}{|c|}{ Cumulative renal function rate $(\%)$} & \multicolumn{5}{|c|}{ Renal function year } \\
\hline & $1 \mathrm{yr}$ & $3 \mathrm{yr}^{*}$ & $5 \mathrm{yr}$ & $7 \mathrm{yr}$ & $9 \mathrm{yr}$ & $10 \mathrm{yr}$ & Mean & SD & Median & $\mathrm{SD}$ & $p$ value \\
\hline Non-Infectious & 88.9 & 84.7 & 71.3 & 55.4 & 52.2 & 36.1 & 8.95 & 1.04 & 10.00 & 1.38 & 0.206 \\
\hline Infectious & 81.3 & 56.3 & 50.0 & 37.5 & 31.3 & 31.3 & 6.19 & 1.16 & 6.00 & 3.33 & \\
\hline
\end{tabular}

Non-Infectious, $\mathrm{n}=46$; Infectious, $\mathrm{n}=16$.

$* p<0.05$ 
Table 3. Comparison of immunosuppressive regimens for renal function at different diarrhea periods after kidney transplant

\begin{tabular}{|c|c|c|c|c|c|c|c|c|c|c|c|c|}
\hline \multirow{2}{*}{\multicolumn{2}{|c|}{$\begin{array}{l}\text { Regimen } \\
\mathrm{N}=62\end{array}$}} & \multicolumn{6}{|c|}{ Cumulative renal function rate $(\%)$} & \multicolumn{5}{|c|}{ Renal function year } \\
\hline & & $1 \mathrm{yr}$ & $3 \mathrm{yr}$ & $5 \mathrm{yr}$ & $7 \mathrm{yr}$ & $9 \mathrm{yr}$ & $10 \mathrm{yr}$ & Mean & SD & Median & SD & $p$ value \\
\hline TM & $\mathrm{n}=27$ & 88.9 & 77.8 & 58.3 & 46.3 & 37.4 & 25.0 & 6.9 & 0.8 & 7.0 & 1.5 & 0.207 \\
\hline $\mathrm{CM}$ & $\mathrm{n}=21$ & 85.0 & 70.6 & 63.5 & 63.0 & 63.0 & 37.8 & 7.6 & 1.0 & 10.0 & 3.7 & \\
\hline $\mathrm{mM}$ & $\mathrm{n}=14$ & 85.7 & 85.7 & 85.7 & 54.5 & 54.5 & 54.5 & 10.2 & 1.7 & 11.0 & 2.8 & \\
\hline
\end{tabular}

TM=TAC-MMF

$\mathrm{CM}=\mathrm{CsA}-\mathrm{MMF}$

$\mathrm{mM}=\mathrm{mTOR}$ inhibitor-MMF. 
Medical Research Archives, Vol. 4, Issue 7, November 2016

Persistent Diarrhea Impairs Renal Function

Table 4. Independent predictors of deterioration of renal function

\begin{tabular}{llccc}
\hline & & HR & $95 \%$ CI & $p$ value \\
\hline Age & & 1.00 & $0.96-1.04$ & 0.900 \\
Gender & Male & Reference & & \\
& Female & 0.54 & $0.16-1.82$ & 0.318 \\
Infectious & No & Reference & & \\
& Yes & 3.24 & $1.05-10.05$ & 0.041 \\
IS regimens & $\mathrm{mM}$ & Reference & & \\
& $\mathrm{CM}$ & 1.43 & $0.29-7.17$ & 0.663 \\
& $\mathrm{TM}$ & 2.13 & $0.41-11.08$ & 0.367 \\
Diarrhea period & $>10.5$ days & & & \\
\hline
\end{tabular}

IS regimens: Immunosuppressive regimes 


\section{References}

[1] F. Gonzalez, Valjalo R., Combining cytochrome P-450 3A4 modulators and cyclosporine or everolimus in transplantation is successful., World J Transplant. 5 (2015) 338-347. doi:10.5500/wjt.v5.i4.338.

[2] L. Fu, Huang Z., Song T., He S., Zeng D., Rao Z., et al., Short-term therapeutic drug monitoring of mycophenolic acid reduces infection: a prospective, single-center cohort study in Chinese living-related kidney transplantation., Transpl Infect Dis. 16 (2014) 760-766. doi:10.1111/tid.12275.

[3] M. J. Felix, Felipe C. R., TedescoSilva H., Osmar Medina-Pestana J., Time-Dependent and Immunosuppressive DrugAssociated Adverse Event Profiles in De Novo Kidney Transplant Recipients Converted from Tacrolimus to Sirolimus Regimens., Pharmacotherapy. 36 (2016) 152-165. doi:10.1002/phar.1692.

[4] Y. J. Zhao, Wen J. Q., Cheng K., Ming Y. Z., She X. G., Liu H., et al., Late, severe, noninfectious diarrhea after renal transplantation: high-risk factors, therapy, and prognosis., Transplant Proc. 45 (2013) 22262232 .

doi:10.1016/j.transproceed.2013.02.1 31.

[5] E. Savvidaki, Papachristou E., Kazakopoulos P., Papasotiriou M., Vardoulaki M., Goumenos D. S., Gastrointestinal disorders after renal transplantation., Transplant Proc. 46 (2014)

3183-3186. doi:10.1016/j.transproceed.2014.09.1 55.

[6] B. Maes, Hadaya K., de Moor B., Cambier P., Peeters P., de Meester J., et al., Severe diarrhea in renal transplant patients: results of the DIDACT study., Am J Transplant. 6 (2006) 1466-1472. doi:10.1111/j.16006143.2006.01320.x.

[7] S. Brakemeier, Taxeidi S. I., Durr M., Hofmann J., Schmidt D., Bachmann F., et al., Clinical outcome of norovirus infection in renal transplant patients., Clin Transplant. (2016) doi:10.1111/ctr.12820. 
[8] K. Raja, Abbas Z., Hassan S. M., Luck N. H., Aziz T., Mubarak M., Prevalence of cryptosporidiosis in renal transplant recipients presenting with acute diarrhea at a single center in Pakistan., J Nephropathol. 3 (2014) 127-131. doi:10.12860/jnp.2014.25.

[9] T. S. Altıparmak MR, Pamuk O“ N, Apaydın S, Sarıyar M, O” ztu“ rk R, Ataman R S. t. K., Erek E, Diarrhoea following renal transplantation., Clin Transplant. 16 (2002) 212-216.

[10] H. Y. Wang, Yeh M. K., Tian Y. F., Huang Y. B., Effect of Prolonged Diarrhea in Renal Transplant Recipients at a Single Center in Taiwan., Transplant Proc. 48 (2016) 870-873.

doi:10.1016/j.transproceed.2015.12.0 75 S0041-1345(16)00173-1 [pii].

[11] G. Guerra, Ciancio G., Gaynor J. J., Zarak A., Brown R., Hanson L., et al., Randomized trial of immunosuppressive regimens in renal transplantation., J Am Soc Nephrol. 22 (2011) 1758-1768. doi:10.1681/ASN.2011010006 ASN.2011010006 [pii].

[12] M. Angarone, Ison M. G., Diarrhea in solid organ transplant recipients. Curr Opin Infect Dis. 28 (2015) 308316.

10.1097/QCO.0000000000000172.

[13] H. Arslan, Inci E. K., Azap O. K., Karakayali H., Torgay A., Haberal M., Etiologic agents of diarrhea in solid organ recipients., Transpl Infect Dis. 9 (2007) 270-275. doi:10.1111/j.13993062.2007.00237.x.

[14] G. Bamias, Boletis J., Argyropoulos T., Skalioti C., Siakavellas S. I., Delladetsima I., et al., Early ileocolonoscopy with biopsy for the evaluation of persistent posttransplantation diarrhea., World J Gastroenterol. 16 (2010) 3834-3840. http://www.ncbi.nlm.nih.gov/pubme $\mathrm{d} / 20698047$.

[15] H. Ekberg, Kyllonen L., Madsen S., Grave G., Solbu D., Holdaas H., Increased prevalence of gastrointestinal symptoms associated with impaired quality of life in renal transplant recipients., Transplantation. 83 (2007) 282-289. doi:10.1097/01.tp.0000251923.1469 7.f5 00007890-200702150-00010 
[pii].

[16] S. Bunnapradist, Neri L., Wong W., Lentine K. L., Burroughs T. E., Pinsky B. W., et al., Incidence and risk factors for diarrhea following kidney transplantation and association with graft loss and mortality., Am J Kidney Dis. 51 (2008) 478-486. doi:10.1053/j.ajkd.2007.11.013 S0272-6386(07)01587-9 [pii].

[17] R. S. Gaston, Kaplan B., Shah T., Cibrik D., Shaw L. M., Angelis M., et al., Fixed- or controlled-dose mycophenolate mofetil with standard- or reduced-dose calcineurin inhibitors: the Opticept trial., Am J Transplant. 9 (2009) 1607-1619.

doi:10.1111/j.1600-6143.2009.02668.x

\section{AJT2668 [pii].}

[18] S. Gil-Vernet, Amado A., Ortega F., Alarcon A., Bernal G., Capdevila L., et al., Gastrointestinal complications in renal transplant recipients: MITOS study., Transplant Proc. 39 (2007) 2190-2193. doi:S00411345(07)00804-4 [pii] 10.1016/j.transproceed.2007.07.015.
[19] K. Boudjema, Camus C., Saliba F., Calmus Y., Salame E., Pageaux G., et al., Reduced-dose tacrolimus with mycophenolate mofetil vs. standarddose tacrolimus in liver transplantation: a randomized study., Am J Transplant. 11 (2011) 965-976. doi:10.1111/j.16006143.2011.03486.x.

[20] C. Pant, Deshpande A., Larson A., O'Connor J., Rolston D. D., Sferra T. J., Diarrhea in solid-organ transplant recipients: a review of the evidence., Curr Med Res Opin. 29 (2013) 13151328.

doi:10.1185/03007995.2013.816278.

[21] N. M. Davies, Grinyo J., Heading R., Maes B., Meier-Kriesche H. U., Oellerich M., Gastrointestinal side effects of mycophenolic acid in renal transplant patients: a reappraisal., Nephrol Dial Transplant. 22 (2007) 2440-2448. doi:10.1093/ndt/gfm308.

[22] M. Behrend, Adverse gastrointestinal effects of mycophenolate mofetil: aetiology, incidence and management., Drug Saf. 24 (2001) 645-663.

[23] A. Aiyangar, Rajput P., Shah B. V., 
Mycophenolate induced diarrhoea., J

Assoc Physicians India. 58 (2010) 192-194.

[24] A. Husing, Schmidt M., Beckebaum

S., Cicinnati V. R., Koch R.,

Tholking G., et al., Long-Term Renal
Function in Liver Transplant

Recipients After Conversion From

Calcineurin Inhibitors to mTOR

Inhibitors., Ann Transplant. 20 (2015)

707-713. doi:895320 [pii]. 